\title{
Laboreal
}

Volume $1 \mathbf{N}^{\circ} 1$ | 2005

Varia

\section{El proyecto de una sociedad del conocimiento : de Lev Vygotski a prácticas efectivas de formación continua en Portugal}

0 projecto de uma sociedade do conhecimento : de Lev Vygotski a práticas efectivas de formação contínua em Portugal

Le projet d'une société de la connaissance : de Lev Vygotski aux pratiques

effectives de formation continue au Portugal

The project of a knowledge society : from Lev Vygotski to continuous training

effective practices in Portugal

\section{Marta Santos}

\section{OpenEdition}

\section{Journals}

\section{Edición electrónica}

URL: http://journals.openedition.org/laboreal/14092

DOI: $10.4000 /$ laboreal. 14092

ISSN: 1646-5237

\section{Editor}

Universidade do Porto

Referencia electrónica

Marta Santos, «El proyecto de una sociedad del conocimiento : de Lev Vygotski a prácticas efectivas de formación continua en Portugal », Laboreal [En línea], Volume 1 Nº1 | 2005, Publicado el 01 diciembre 2005, consultado el 24 septiembre 2020. URL : http://journals.openedition.org/laboreal/ 14092 ; DOI : https://doi.org/10.4000/laboreal.14092

Este documento fue generado automáticamente el 24 septiembre 2020

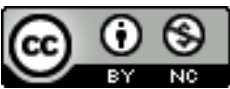

Laboreal está licenciado com uma Licença Creative Commons - Atribuição-NãoComercial 4.0 Internacional. 


\section{El proyecto de una sociedad del conocimiento : de Lev Vygotski a prácticas efectivas de formación continua en Portugal}

O projecto de uma sociedade do conhecimento : de Lev Vygotski a práticas efectivas de formação contínua em Portugal

Le projet d'une société de la connaissance : de Lev Vygotski aux pratiques

effectives de formation continue au Portugal

The project of a knowledge society: from Lev Vygotski to continuous training

effective practices in Portugal

\section{Marta Santos}

\section{REFERENCIA}

Santos, M. (2004). O projecto de uma sociedade do conhecimento : de Lev Vygotski a práticas efectivas de formação contínua em Portugal. Tese de Doutoramento, Faculdade de Psicologia e Ciências da Educação da Universidade do Porto, Porto.

\section{NOTA DEL EDITOR}

Manuscrito recibido en: julio/2005

Aceptado tras peritage en: noviembre/2005 


\section{Punto de partida}

1 El punto de partida para este trabajo fue la constatación, desde el análisis de discursos oficiales portugueses y al nivel de la Unión Europea, de que se deposita en la construcción de una sociedad del conocimiento la esperanza de una nueva armonía social, que se alcanzaría gracias al alargamiento y generalización del acceso a la educación y formación a lo largo de la vida. Conociendo prácticas formativas muy variables en lo que se refiere a la forma como se conciben y concretan (objetivos, contenidos, metodologías y actores de formación), hemos formulado como objetivo de este estudio la comprensión de la articulación de estos discursos con las acciones de formación reales que acabamos por encontrar. Lo que pretendíamos era contraponer los grandes principios generales al análisis de prácticas reales de forma a delinear lo que es posible, lo que acaba por hacerse, lo que podría haberse hecho, lo que no se tiene en cuenta.

2 Paralelamente, asistimos a un interés creciente por la obra de Vygotski (1997). Preocupado con el aparecimiento de las funciones psicológicas superiores, específicamente humanas, este autor expuso una teoría que sugiere que determinadas intervenciones formativas, conducidas en articulación con la especificidad del recorrido histórico del individuo, permiten el desarrollo a los adultos.

3 Sin embargo, Vygotski no se ha inclinado específicamente sobre las situaciones de formación profesional por lo que creemos interesante comprender este cuadro teórico de base por la referencia a la aportación de 3 autores contemporáneos que lo han prolongado en el estudio de esta área.

4 Así, en este trabajo, procuramos articular las perspectivas teóricas desarrolladas por Clot (1999), Rabardel (1995) y Pastré (1999, 2002), elegidos como protagonistas de este discurso científico, con el análisis de ciertos aspectos de 3 acciones de formación continua.

\section{Tres autores, tres acciones}

5 En lo que se relaciona con Clot, hemos retenido la importancia de la (re)construcción del género profesional las reglas y normas de la profesión compartidas y validadas por un colectivo de trabajo por permitir el reconocimiento de la experiencia y la atribución de un sentido para el trabajo. Aquí, el trabajo del investigador/formador corresponde a la participación, con su interpretación de la situación, en un proceso de co-análisis del trabajo.

6 Relativamente a una de las tres acciones de formación (que transcurrió en una empresa de tejedura), procuramos verificar la posibilidad de aplicación de los principales conceptos de este autor.

7 Se comprobó que, en el ámbito del proyecto de una sociedad del conocimiento, es posible sacar partido de momentos de discusión colectiva para la construcción de nuevas representaciones sobre las situaciones de trabajo. Pero se mostró también que conviene estar atento al hecho de que esta sociedad negligencia, de forma tendenciosa, el cuanto la actividad cognitiva se ancla en los registros del cuerpo, siendo esos frecuentemente entendidos, en el lugar de trabajo, como "barreras" al desarrollo de la lógica de la empresa y, por eso, no valorados. 
8 Relativamente al segundo cuadro teórico a que recurrimos Rabardel pensamos que su principal aportación se debe al desarrollo de la noción de instrumento, que es entendido como una entidad mixta que reúne en si un artefacto (material o simbólico) y los esquemas que permiten su uso. Esto significa que el artefacto sólo se convierte en un verdadero instrumento cuando se inscribe en un uso, cuando es un medio para el usuario poder realizar un determinado objetivo, es decir, a través de procesos de génesis instrumental.

9 Hemos utilizado este cuadro teórico como cuadro de lectura de una acción de formación en un almacén de papel, revelando que la distancia entre lo que se proponía a hacer y lo que realmente acaba por ser implementado se debe, entre otras, a presiones temporales, determinadas por condicionalismos económicos poco sensibles a las condiciones efectivamente necesarias para el concretar del proyecto de una sociedad del conocimiento.

10 Cuanto al tercer cuadro teórico, podemos decir que la especificidad de la obra de Pastré ha incidido en las competencias críticas que son desarrolladas por los trabajadores: procura la identificación de los conceptos pragmáticos movilizados por los especialistas en las situaciones de trabajo, de forma a identificar su estructura conceptual, que es aquella que retiene apenas lo que se mantiene invariante.

11 Este abordaje ha sostenido el seguimiento de una acción de formación para contramaestres pescadores. Articulada sobre aportaciones que conciben el desarrollo cognitivo como el acceso a la acción eficaz, el análisis de esta formación ha permitido definir mejor las dificultades de las personas en formación en organizar su experiencia y sus discursos en función de conceptos invariantes. Por otro lado, los retos de la sociedad del conocimiento parecen determinar, en estos trabajadores, sentimientos contradictorios entre la voluntad de conocer y dominar el lenguaje de las nuevas tecnologías y el miedo a perder los conocimientos y formas de actuar que poseen y que hasta ahí se habían mostrado adecuados.

\section{Más allá de Vygotski}

12 Asociando estas reflexiones con la pertinencia de la obra de Vygotski para la concepción de acciones de formación continua, y partiendo de su prolongamiento en los cuadros teóricos de los tres autores contemporáneos, pensamos que es en clot que se nota, de forma más estructural, la filiación en la perspectiva histórico-cultural de Vygotski. Sin embargo Clot propone un desdoblamiento del concepto de zona de desarrollo proximal de Vygotski: más allá del desarrollo cognitivo, es importante intervenir en el desarrollo de los motivos de las actividades.

Rabardel, prolonga Vygotski a partir del concepto de génesis instrumental, considerando que, para que se adquiera un instrumento además de una representación sobre él, es necesaria la posibilidad de utilizarlo.

14 Pastré también lo prolonga, sobretodo a partir de la noción de concepto pragmático, que comporta elementos para conceptualizar, pero que se distingue de los conceptos científicos por su validad local.

15 Pero las propuestas de estos autores contemporáneos deben también ser asociadas al tipo de pedidos a los que se intenta dar respuesta y a la forma como elaboran sus intervenciones. Así, mientras que los abordajes de Pastré y Rabardel se preocupan 
particularmente con los momentos en los que hay cambios en las situaciones de trabajo, procurando concebir situaciones de aprendizaje que permitan la actualización de competencias y la adaptación a las nuevas situaciones (y en este sentido parecen más próximos del tipo de proyectos enunciados por la sociedad del conocimiento); por su lado Clot, parece estar más preocupado en dar visibilidad al trabajo como potencial de desarrollo y, también, como fuente de sufrimiento e infiabilidad.

\section{Vygotski en (des)uso en la sociedad del conocimiento}

16 En lo que concierne al cuadro teórico propuesto por Vygotski, nos gustaría reforzar su importancia al abrir la posibilidad para un "desarrollo a lo largo de la vida". La aplicación de este principio a la formación profesional continua, daría con toda la seguridad origen a acciones de formación que funcionasen como el aprendizaje de un idioma extranjero: es un hecho que no son raros los casos en los que se realizan las actividades de trabajo de forma muy similar a la que se utiliza con el lenguaje materno. Se aprende en el día-a-día, observando, ensayando: se sabe lo que se realiza pero no siempre con la conciencia de la forma como lo es. Una acción de formación continua que visase el desarrollo de las personas en formación tendría, entonces, que actuar como si del aprendizaje de un idioma extranjero se tratara, teniendo como consecuencia la posibilidad de pasar a tomar conciencia del sistema simbólico utilizado en la actividad de trabajo, es decir, hacer del trabajo un objeto de reflexión. De este modo, uniendo una forma de pensamiento no verbal al lenguaje, pasaríamos para el dominio del pensamiento verbal, conceptual, reflexionando, consciente y, entonces, promotor de desarrollo "integral y armonioso".

17 Puesta así la cuestión, nos quedamos con la idea de que, para Vygotski, existe un a priori del que todos los nuevos conocimientos, adquiridos por intermedio de una situación de cooperación asimétrica, son buenos, permiten nuestro desarrollo. Esto significa que son sobretodo los conceptos científicos, mediados por el lenguaje, que permiten el pensamiento verbal, que son valorados.

18 No cuestionamos la importancia para el desarrollo humano de la adquisición del pensamiento verbal, pero ¿será que todos los nuevos conocimientos adquiridos se muestran más eficaces?

19 Creer que el acceso a los nuevos conocimientos, por si sólo, es positivo, no permite entender el miedo a perder lo que se sabe y se muestra eficaz (situación que hemos encontrado en el momento del análisis de la acción de formación para contramaestres pescadores).

20 Así, pensamos que los nuevos conocimientos sólo son benéficos cuando son ponderados en relación a los conocimientos que ya se tienen, pudiendo funcionar como un recurso alternativo o complementario.

21 Concluyendo, podemos decir que, en el discurso sobre la sociedad del conocimiento, se enuncian medidas que visan reducir las desigualdades por el acceso al conocimiento. Sin embargo, hemos llegado a un conjunto de conclusiones que cuestionan fundamentalmente la mayoría de las prácticas hasta ahora desarrolladas:

- el acceso a las acciones de formación está condicionado por dinámicas sociales que acaban por marginalizar a los ciudadanos que más necesitan de ellas; 
- por la forma como transcurren, no todas las acciones producen los efectos intentados;

- hay determinados conocimientos que son considerados a priori más eficaces o mejores que los demás: parecen existir los conocimiento de "arriba", de una sociedad del conocimiento, incuestionables, considerados como fuente de progreso; y, los conocimientos de "abajo", obsoletos, desactualizados, poseídos por las personas menos cualificadas y que "son trabas" al desarrollo de la sociedad. Además, la ausencia, en las acciones de formación observadas, de condiciones para un debate contradictorio entre los diferentes tipos de conocimientos refuerza esta contradicción interna del proyecto de la sociedad del conocimiento.

\section{BIBLIOGRAFÍA}

Clot, Y. (1999). La fonction psychologique du travail. Paris: PUF.

Pastré, P. (1999). La conceptualisation dans l'action: bilan et nouvelles perspectives. Education Permanente, 139, 2, 13-35.

Pastré, P. (2002). L'analyse du travail en didactique professionnelle. Revue Française de Pédagogie, 138, 9-17.

Rabardel, P. (1995). Les hommes et les technologies: approche cognitive des instruments contemporains. Paris: Armand Colin.

Vygotski, L. (1997). Pensée \& langage. (3e édition) (F. Séve, trad.). Paris: La Dispute. (Edição original, 1934).

\section{AUTOR}

\section{MARTA SANTOS}

Centro de Psicologia da Universidade do Porto - Faculdade de Psicologia e de Ciências da Educação da Universidade do Porto Rua do Campo Alegre, 1055, 4169-004 Porto, Portugal marta@fpce.up.pt 